\title{
Effect of a cholesterol-rich diet on the metabolism of the free and esterified cholesterol components of a nanoemulsion that resembles $L D L$ in rabbits
}

\author{
A.F. Padoveze1, F. Maniero', T.V. Oliveira1, T.S. Vitorio², R.D. Couto ${ }^{1,3}$ and \\ R.C. Maranhão ${ }^{1,2}$
}

${ }^{1}$ Laboratório de Metabolismo de Lípides, Instituto do Coração, Hospital das Clínicas, Faculdade de Medicina, Universidade de São Paulo, São Paulo, SP, Brasil

2Departamento de Análises Clínicas e Toxicológicas, Faculdade de Ciências Farmacêuticas, Universidade de São Paulo, São Paulo, SP, Brasil

${ }^{3}$ Departamento de Análises Clínicas e Toxicológicas, Faculdade de Farmácia, Universidade Federal da Bahia, Salvador, BA, Brasil

Correspondence to: R.C. Maranhão, Laboratório de Metabolismo de Lípides, InCor, HC, FM, USP, Av. Dr. Enéas C. Aguiar, 44, Bloco II, $1^{\circ}$ Subsolo, 05403-000 São Paulo, SP, Brasil

Fax:+55-11-3069-5574. E-mail: ramarans@usp.br

\begin{abstract}
We have shown that the free cholesterol (FC) and the cholesteryl ester (CE) moieties of a nanoemulsion with lipidic structure resembling low-density lipoproteins show distinct metabolic fate in subjects and that this may be related to the presence of dyslipidemia and atherosclerosis. The question was raised whether induction of hyperlipidemia and atherosclerosis in rabbits would affect the metabolic behavior of the two cholesterol forms. Male New Zealand rabbits aged 4-5 months were allocated to a control group $(\mathrm{N}=17)$ fed regular chow and to a $1 \%$ cholesterol-fed group $(\mathrm{N}=13)$ during a 2-month period. Subsequently, the nanoemulsion labeled with ${ }^{3} \mathrm{H}-\mathrm{FC}$ and ${ }^{14} \mathrm{C}-$ $\mathrm{CE}$ was injected intravenously for the determination of plasma kinetics and tissue uptake of the radioactive labels. In controls, FC and CE had similar plasma kinetics (fractional clearance rate, $F C R=0.234 \pm 0.056$ and $0.170 \pm 0.038 \mathrm{~h}^{-1}$, respectively; $\mathrm{P}=0.065$ ). In cholesterolfed rabbits, the clearance of both labels was delayed and, as a remarkable feature, FC-FCR $\left(0.089 \pm 0.033 \mathrm{~h}^{-1}\right)$ was considerably greater than CE-FCR $\left(0.046 \pm 0.010 \mathrm{~h}^{-1} ; \mathrm{P}=0.026\right)$. In the liver, the major nanoemulsion uptake site, uptake of the labels was similar in control animals ( $F C=0.2256 \pm 0.1475$ and $C E=0.2135 \pm 0.1580 \% / g)$ but in cholesterol-fed animals $F C$ uptake $(0.0890 \pm 0.0319 \% / g)$ was greater than CE uptake $(0.0595 \pm 0.0207 \% / g ; P<0.05)$. Therefore, whereas in controls, FC and CE have similar metabolism, the induction of dyslipidemia and atherosclerosis resulted in dissociation of the two forms of cholesterol.
\end{abstract}

Key words: Lipoprotein kinetics; Free cholesterol; Hyperlipidemia; Rabbitatherosclerosis; Nanoemulsions; Cholesterol-rich diet

Research supported by FAPESP (\#04/08048-3). R.C. Maranhão was the recipient of a Research Award from CNPq.

Received May 4, 2008. Accepted January 15, 2009

\section{Introduction}

Artificial lipid nanoemulsions can be used as probes to detect genetically inherited alterations or evaluate pharmacological, nutritional and other interventions on lipoprotein metabolism (1-4). A single preparation of these artificial nanoemulsions can be injected into several pa- tients for plasma kinetic studies, whereas only the autologous native lipoprotein can be injected into subjects, due to the risk of infection or immunological reactions resulting from the use of heterologous lipoproteins. In addition, natural lipoproteins are difficult to isolate and label with radioactive or stable isotopes or other means, difficulties that are easily overcome in the preparation of 
artificial nanoparticles. Nanoparticles can also be engineered to be more rapidly removed from the circulation, such that the observation time required for the clearance test is substantially shortened (5).

In previous studies, we showed that the removal from the plasma of the free cholesterol (FC) label of a nanoemulsion that probes the low-density lipoprotein (LDL) metabolism was faster in coronary artery disease (CAD) patients than in non-CAD control subjects, whereas the removal of the nanoemulsion cholesteryl esters (CE) was similar in both groups (6). The dissociation of the plasma kinetics of the two forms of cholesterol was also documented in our recent study involving patients with familial hypercholesterolemia (7). As expected, the removal of the nanoemulsion CE was delayed in the hypercholesterolemic patients, as occurs with the kinetics of apolipoprotein (apo) B of native LDL under these conditions. As to the removal of $\mathrm{FC}$ it was equal to that of $\mathrm{CE}$ but its reduction in familial hypercholesterolemic patients was not as marked as that of CE. On the other hand, whereas the treatment with simvastatin normalized the removal of CE, FC was accelerated but at lower rates (7). Those findings support the view that the two forms of cholesterol composing the structure of the same lipoprotein particles may undergo different metabolic fates. The double-labeled nanoemulsion was also injected into CAD patients who were scheduled for coronary artery bypass grafting and it was shown that whereas in the plasma there were more CE than FC, in vessel fragments from the radial, femoral, aorta, and saphenous vein the uptake of FC was greater than that of CE (8). The results suggest that FC molecules may dissociate from the nanoemulsion particles, leading to deposition of FC in the vessel wall. As the nanoemulsion mimicks LDL, this phenomenon may also occur with FC of the native lipoprotein.

To further explore these metabolic divergences between FC and CE, we determined whether induction of hyperlipidemia and atherosclerosis in animals would give rise to alterations linked with the dissociation of the metabolism of the two cholesterol forms. Thus, we determined the plasma kinetics and the uptake by several tissues of the two forms of cholesterol contained in the nanoemulsion injected into control rabbits and rabbits fed a cholesterol-rich diet for 2 months.

\section{Material and Methods}

\section{Animals and diets}

Thirty male New Zealand white rabbits weighing $2.6 \pm$ $0.2 \mathrm{~kg}$ (mean $\pm \mathrm{SD}$ ) were housed in individual cages in a temperature-controlled room $\left(20-22^{\circ} \mathrm{C}\right)$, on a 12-h light/ dark cycle. After a 1-week adaptation period, the rabbits were separated into two groups. The control group was fed regular laboratory rabbit chow (Nuvital Nutrientes S/A, Brazil) for 8 weeks. The cholesterol-fed group received an atherogenic diet consisting of chow enriched with $1 \%$ (w/w) cholesterol (Dolder AG, Switzerland) for the same period. The amount of daily diet ingested by each animal was restricted to $150 \mathrm{~g}$ and was controlled by weighing the non-eaten chow every 4 days. Water was available ad libitum. There were no changes in food intake by the two groups during the experimental period. The body weight was recorded every 8 days. After 5 -week cholesterol feeding, animal body weight increased $15 \pm 1 \%$. This study conformed to the guidelines for the care and use of animals as approved by the Ethics Committee of the Instituto do Coração, Hospital das Clínicas, Universidade de São Paulo.

\section{Lipid analysis}

Blood samples were withdrawn from the marginal ear vein of the rabbits after overnight fasting before and after the study period. Serum was obtained by centrifugation at $1200 \mathrm{~g}$ for $10 \mathrm{~min}$ at $4^{\circ} \mathrm{C}$. Total cholesterol, high-density lipoprotein (HDL) cholesterol, and triglycerides were determined by commercial kits obtained from Labtest Diagnostica S.A. (Brazil). Non-HDL cholesterol was calculated as the difference between total and HDL-cholesterol. Results are reported as means of duplicate samples.

\section{Preparation of the labeled artificial nanoemulsion}

The artificial nanoemulsion was prepared as described previously by Maranhão et al. (4), with addition of $\left[1-{ }^{14} \mathrm{C}\right]$ cholesteryl oleate and $\left[7(\mathrm{n}){ }^{-3} \mathrm{H}\right]$ cholesterol (Amersham International, England) and was sterilized by passage through a $0.22-\mu \mathrm{m}$ filter for use in animal experiments.

\section{Plasma kinetics}

The nanoemulsion containing ${ }^{3} \mathrm{H}-\mathrm{FC}(0.8 \mu \mathrm{Ci})$ and ${ }^{14} \mathrm{C}$-CE $(0.6 \mu \mathrm{Ci})$ was injected into the marginal ear vein of rabbits after 8 weeks of feeding on the regular or atherogenic diet. Plasma samples were collected from the contralateral ear vein $5 \mathrm{~min}, 1,2,4,6,8$, and $24 \mathrm{~h}$ after the injection to evaluate the plasma kinetics and the fractional clearance rates (FCR) of the radioactive lipids. Aliquots $(100 \mu \mathrm{L})$ of blood plasma were transferred to counting vials containing $5.0 \mathrm{~mL}$ of scintillation solution (Ultima Gold XR, Perkin Elmer, USA) and the radioactivity was counted using a Packard 1660 TR (USA) spectrometer.

FCR of the ${ }^{3} \mathrm{H}-\mathrm{FC}$ and ${ }^{14} \mathrm{C}-\mathrm{CE}$ labels were calculated from biexponential curves obtained from the remaining 
radioactivity found in plasma after injection as described previously (9), using the ANACOMP software for compartmental analysis (10).

\section{Nanoemulsion uptake by body tissues}

Rabbits were killed by intravenous injection of a lethal dose of sodium pentobarbital (5\%) $24 \mathrm{~h}$ after the injection of artificial labeled nanoemulsion and their organs (liver, lungs, heart, spleen, kidney, pancreas, gonads, adrenals, gallbladder, skin, adipose and skeletal muscle tissues, and aorta segments) were excised and rinsed in ice-cold $0.9 \% \mathrm{NaCl}$. Fragments of organs and tissues were then chopped and lipids from 150-500 mg tissues were extracted with chloroform/methanol $(2: 1, \mathrm{v} / \mathrm{v})(11)$ and the radioactivity was measured in a scintillation solution (UItima Gold XR, Perkin Elmer).

\section{Statistical analysis}

Results are reported as means \pm SD. Differences in the FCR of FC and CE and tissue uptake between the groups were assessed using the Mann-Whitney nonparametric test. Differences in total cholesterol, HDL cholesterol, non-HDL cholesterol and triglycerides were assessed using the paired nonparametric Wilcoxon test. Kruskal-Wallis nonparametric ANOVA was used to evaluate the animal's weight body and food intake in the study period, and compare the uptake of FC and CE by several tissues in each group. The correlation between serum lipid concentration and FCR of FC and CE was assessed using the Spearman nonparametric test. In all analyses, two-tailed $\mathrm{P}<0.5$ was considered to be statistically significant. All analyses were performed using the statistical software GraphPad (USA).

\section{Results}

Serum lipids and induction of atherosclerosis

Table 1 shows the concentration of the serum lipids in the cholesterol-fed group before and at the end of the two-month period of cholesterol feeding. Cholesterol feeding increased the values of total and non-HDL cholesterol by roughly 40 - and 70 -fold, respectively, but did not alter the HDL cholesterol. The serum triglyceride values were increased 2-fold by cholesterol feeding. In the group of cholesterol-fed animals, the atherosclerosis process was intense, mainly in the aortic arch, as observed by macroscopic examination, while in the control animals no lesions were observed. Based on microscopic morphometry, the intima/media area ratio was $0.26 \pm 0.06$ in the aortic arch, $0.23 \pm 0.21$ in the thoracic aorta and $0.17 \pm$ 0.16 in the abdominal aorta of the cholesterol-fed rabbits.

\section{Plasma kinetics}

Figure 1 shows the plasma decay curves of $\mathrm{FC}$ and $\mathrm{CE}$ of the artificial nanoemulsion. It is apparent that in the control animals (Panel $A$ ), there was no difference between the decay curves of the two forms of cholesterol. After feeding a cholesterol-rich diet, both decay curves became slower, but FC was removed faster than $\mathrm{CE}$. In fact, in the control group, the FCR of CE and FC were not different $\left(0.234 \pm 0.056\right.$ and $0.170 \pm 0.038 h^{-1}$, respective-

Table 1. Lipid profile of 13 rabbits with induced atherosclerosis at baseline and after cholesterol feeding.

\begin{tabular}{llc}
\hline Lipid profile & Baseline & After feeding \\
\hline Cholesterol $(\mathrm{mg} / \mathrm{dL})$ & & \\
$\quad$ Total & $51 \pm 14$ & $2267 \pm 562^{*}$ \\
HDL & $19 \pm 7$ & $29 \pm 16$ \\
$\quad$ Non-HDL & $32 \pm 2$ & $2310 \pm 547^{*}$ \\
Triglycerides $(\mathrm{mg} / \mathrm{dL})$ & $88 \pm 36$ & $214 \pm 159^{*}$
\end{tabular}

Data are reported as mean $\pm \mathrm{SD}$. $\mathrm{HDL}=$ high-density lipoprotein cholesterol. ${ }^{*} P<0.01$, compared to baseline (Wilcoxon test).

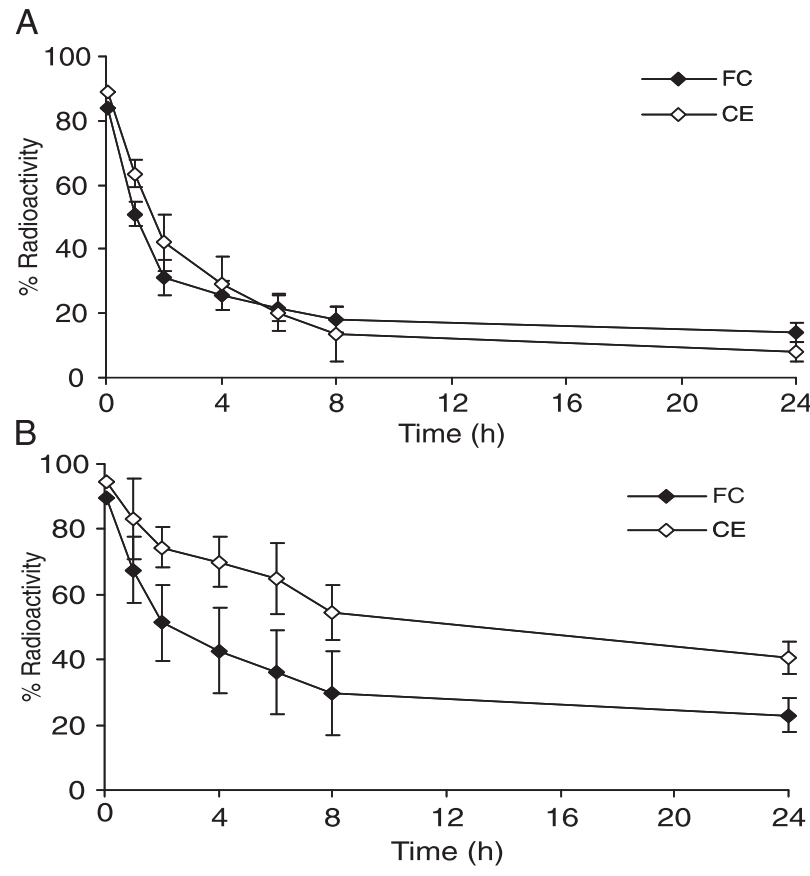

Figure 1. Plasma decay curves of the artificial nanoemulsion ${ }^{3} \mathrm{H}$-free cholesterol (FC) and ${ }^{14} \mathrm{C}$-cholesteryl esters (CE). $A$, Control group $(N=8) ; B$, cholesterol-fed group $(N=6)$. The artificial labeled nanoemulsion was injected into the marginal ear vein of rabbits after 8 weeks feeding of a regular or an atherogenic diet. Plasma samples were obtained at intervals over $24 \mathrm{~h}$ for counting of the radioactivity in a liquid scintillation solution. Data are reported as mean $\pm S D$. 
ly; $P=0.065)$. Both were reduced after cholesterol feeding $(\mathrm{CE}-\mathrm{FCR}=0.046 \pm 0.010, \mathrm{FC}-\mathrm{FCR}=0.089 \pm 0.033$ $\left.\mathrm{h}^{-1}\right)$ and $\mathrm{FC}-\mathrm{FCR}$ became greater than CE-FCR $(\mathrm{P}=0.026)$.

A correlation analysis was carried out on the serum lipid concentration and FCR data obtained from all animals, with control and cholesterol-fed groups pooled. A negative correlation between both FCR of CE and FC and serum total cholesterol was found. The impact of the serum total cholesterol concentration was greater on CEFCR than on FC-FCR, since both the correlation curve incline and fitting were greater in the CE-FCR correlation plot $(r=-0.82, P=0.0019)$ than in the FC-FCR plot $(r=$ $-0.63, P=0.0323)$.

\section{Tissue uptake}

Table 2 shows the uptake of FC and CE of the artificial nanoemulsion by different tissues. In the control group, the uptake of FC was similar to the CE uptake in most tissues (liver, kidney, pancreas, gonads, adrenals, gallbladder, adipose tissue, muscle, skin, and the aorta segments). On the other hand, in the lung, heart and spleen, the uptake of $F C$ was greater than that of CE.

As also shown in Table 2, the tissue uptake of the FC and CE diminished after the 2-month feeding of the animals with cholesterol, which produced intense hyperlipidemia. In this respect, the uptakes of FC and CE by the liver, which is the main organ that removes lipoproteins from the circulation, were reduced 2.6 and 3.6 times, respectively $(P<0.01)$. After cholesterol feeding, the uptake of $\mathrm{FC}$ by the liver and by the kidney became greater than that of CE. In contrast, FC uptake became smaller than CE in the aortic arch, whereas in the other tissues (pancreas, gonads, adrenals, gall bladder, adipose tissue, muscle, skin, and the other aorta segments) there were no differences between FC and CE uptakes.

\section{Discussion}

In this study, the plasma decay curves of the nanoemulsion FC and CE coincide in control rabbits. When the animals were fed a cholesterol-rich diet that induces extreme hyperlipidemia and atherosclerosis, the clearance of the two forms of cholesterol dissociated into two distinct decay curves, the FC curve becoming faster than that of the CE curve. This indicates that cholesterol feeding, by slowing down the removal of the nanoparticles and by other intervening mechanisms, facilitates the leakage of the FC form from the nanoparticles and, by extrapolation, we would also expect from the native lipoproteins such as LDL. As CE remains in the core, it is the marker of the particles; thus, CE removal from the plasma becomes slower compared to FC when the removal of

Table 2. Uptake of ${ }^{3} \mathrm{H}$-free cholesterol and ${ }^{14} \mathrm{C}$-cholesteryl esters by organs and tissues of cholesterol-fed and control rabbits $24 \mathrm{~h}$ after injection of the artificial nanoemulsion.

\begin{tabular}{|c|c|c|c|c|}
\hline & \multicolumn{2}{|c|}{ Control group $(\mathrm{N}=17)$} & \multicolumn{2}{|c|}{ Cholesterol-fed group $(\mathrm{N}=13)$} \\
\hline & ${ }^{3} \mathrm{H}-\mathrm{FC}$ & ${ }^{14} \mathrm{C}-\mathrm{CE}$ & ${ }^{3} \mathrm{H}-\mathrm{FC}$ & ${ }^{14} \mathrm{C}-\mathrm{CE}$ \\
\hline Liver & $0.2256 \pm 0.1475$ & $0.2135 \pm 0.1580$ & $0.0890 \pm 0.0319^{+\ddagger}$ & $0.0595 \pm 0.0207 \S$ \\
\hline Lungs & $0.2498 \pm 0.1513^{*}$ & $0.2047 \pm 0.4415$ & $0.0511 \pm 0.0345^{\ddagger}$ & $0.0290 \pm 0.0158 \S$ \\
\hline Heart & $0.1459 \pm 0.1340^{*}$ & $0.0498 \pm 0.0574$ & $0.0365 \pm 0.0391 \ddagger$ & $0.0272 \pm 0.0317$ \\
\hline Spleen & $0.2943 \pm 0.1620^{*}$ & $0.1139 \pm 0.0843$ & $0.0453 \pm 0.0332 \ddagger$ & $0.0376 \pm 0.0247 \S$ \\
\hline Kidney & $0.1110 \pm 0.0742$ & $0.0849 \pm 0.0692$ & $0.0280 \pm 0.0164^{+\ddagger}$ & $0.0138 \pm 0.0063 \S$ \\
\hline Pancreas & $0.0054 \pm 0.0027$ & $0.0072 \pm 0.0036$ & $0.0071 \pm 0.0067$ & $0.0176 \pm 0.0166$ \\
\hline Gonads & $0.0240 \pm 0.0130$ & $0.0144 \pm 0.0032$ & $0.0057 \pm 0.0031$ & $0.0076 \pm 0.0033$ \\
\hline Adrenals & $0.6580 \pm 0.2174$ & $0.3283 \pm 0.1650$ & $0.0355 \pm 0.0870^{\ddagger}$ & $0.0483 \pm 0.0507$ \\
\hline Gallbladder & $0.2568 \pm 0.1547$ & $0.0638 \pm 0.0183$ & $0.0435 \pm 0.0077$ & $0.0470 \pm 0.0051$ \\
\hline Adipose tissue & $0.0071 \pm 0.0042$ & $0.0890 \pm 0.0051$ & $0.0137 \pm 0.0118$ & $0.0016 \pm 0.0001$ \\
\hline Muscle & $0.0566 \pm 0.0527$ & $0.0387 \pm 0.0391$ & $0.0132 \pm 0.0121 \ddagger$ & $0.0186 \pm 0.0160$ \\
\hline Skin & $0.0125 \pm 0.0026$ & $0.0087 \pm 0.0021$ & $0.0037 \pm 0.0019$ & $0.0065 \pm 0.0018$ \\
\hline Aortic arch & $0.0639 \pm 0.0581$ & $0.0507 \pm 0.0441$ & $0.0323 \pm 0.0113^{+}$ & $0.0707 \pm 0.0234$ \\
\hline Thoracic aorta & $0.1025 \pm 0.0789$ & $0.0704 \pm 0.0927$ & $0.0198 \pm 0.0067 \ddagger$ & $0.0290 \pm 0.0195$ \\
\hline Abdominal aorta & $0.0724 \pm 0.0461$ & $0.1375 \pm 0.1509$ & $0.0195 \pm 0.0087 \ddagger$ & $0.0287 \pm 0.0237 \S$ \\
\hline
\end{tabular}

Data are reported as $\%$ of the total injected radioactivity recovered per gram of tissue (mean $\pm \mathrm{SD}) .{ }^{3} \mathrm{H}-\mathrm{FC}={ }^{3} \mathrm{H}$-free cholesterol; ${ }^{14} \mathrm{C}$ $\mathrm{CE}={ }^{14} \mathrm{C}$-cholesteryl esters. ${ }^{*} \mathrm{P}<0.05$ compared with ${ }^{14} \mathrm{C}$-CE in the control group; ${ }^{+} \mathrm{P}<0.05$ compared with ${ }^{14} \mathrm{C}$-CE in the cholesterolfed group; $\ddagger \mathrm{P}<0.05$ compared with the ${ }^{3} \mathrm{H}-\mathrm{FC}$ uptake of the control group; $\S \mathrm{P}<0.05$ compared with the ${ }^{14} \mathrm{C}$ - $\mathrm{CE}$ uptake of the control group (Mann-Whitney rank sum test). 
the particles is slowed down by induction of hyperlipidemia.

Cholesterol esterification is a fundamental process for the homeostasis of sterol in the organism. In the circulation, this reaction is catalyzed by lecithin cholesterol acyl transferase (LCAT) using apoA1 as co-factor. It occurs mostly in the HDL fraction that contains most of the apoA1 present in the plasma and where LCAT is also present (12-14). In the cytoplasm, esterification of cholesterol is catalyzed by acyl cholesterol acyl transferase (15-17) and $\mathrm{CE}$ is the intracellular storage form of cholesterol. Cholesterol esterification is an obligatory step of cholesterol reverse transport, in which cholesterol is released from the cells and is transported back to the liver and, thereafter, excreted to the intestine (18). FC is located in the surface layer of the lipoprotein particles and, thus, it can easily diffuse to the surrounding aqueous medium. The in vitro study by Lundberg and Suominen (19) showed that FC may detach from LDL particles and deposit in cellular membranes of fibroblasts. In contrast, the newly esterified molecules are displaced to the lipoprotein core where they are sequestered from the plasma (20). Thereafter, CE can be dislocated from the lipoprotein particle only by the action of transfer proteins that may shuttle the molecules to other lipoprotein particles (21). Esterification is therefore crucial for the stabilization of the cholesterol pool. Defects in this process, such as those resulting from alterations in the LCAT and apoA1 structures and expression, may result in premature atherosclerosis and tissue deposition of FC such as in xanthomatosis (14, 15,22). It is worthwhile to point out that Hoeg et al. (23) found that overexpression of LCAT in transgenic rabbits and mice (24) prevented the development of atherosclerosis induced by a cholesterol-rich diet $(25,26)$.

Upon entry into the plasma compartment, the artificial LDL-like nanoemulsion that is made without protein acquires several exchangeable apolipoproteins that are bound to the native lipoproteins or are free in the plasma (27). Among those, apoA1 stimulates LCAT for esterification of cholesterol (12) and apoE mediates the binding of the nanoparticles to cell surface lipoprotein receptors, mainly the LDL receptor that internalizes the nanoparticles through a receptor-mediated endocytosis pathway (27) as occurs with the native lipoproteins $(28,29)$.

In the present study, cholesterol feeding led to reduced plasma clearance and there was a negative correlation between serum cholesterol and FCR of both free and esterified cholesterol. The reduction of the clearance was the result of the diminished liver uptake of the labeled nanoemulsion that occurred in cholesterol-fed animals. Because the LDL-like nanoparticles are removed by the LDL receptors (27), it is conceivable that in cholesterol-fed animals the competition for the receptors of the artificial nanoparticles with the natural lipoproteins is increased and thus the removal from the plasma, i.e., the uptake by the tissues, is diminished. Furthermore, cholesterol feeding down-regulates the lipoprotein uptake by the LDL receptors (30-32), which aggravates the competition for receptor binding and makes the internalization of the nanoparticles into the cells more difficult.

In control animals, the plasma decay curves of the nanoemulsion FC and CE were similar, indicating that FC does not dissociate from the nanoparticles while in the plasma. In the liver tissue of the control rabbits, the uptake rates of the two forms of cholesterol were also similar, which implies that they were internalized simultaneously into the cells. In contrast, in animals with induced dyslipidemia and atherosclerosis, while the removal of FC from the plasma became greater than that of $\mathrm{CE}$, the liver uptake of FC was also greater than that of CE. These results suggest that the prolonged circulation time of nanoparticles that facilitated the dissociation of FC from the nanoparticles also led to greater deposition of the dissociated FC in the liver compared to $\mathrm{CE}$, the marker of the removal of the artificial LDL-like nanoemulsion. Upon induction of dyslipidemia and atherosclerosis, the uptake of the nanoemulsion was also reduced in most tissues other than the liver because of the increase in competition with the native lipoproteins for cell uptake.

In the liver, the main uptake organ for VLDL, LDL and lipid nanoparticles, the hyperlipidemia effect on uptake has been well documented (30-32). Nonetheless, it is interesting to point out that in the aortic arch, the main site of atherosclerotic plaque installation $(33,34)$, and also in pancreatic, gonad, gallbladder, skin, and adipose tissue, the induction of hyperlipidemia did not diminish the uptake of the nanoparticle labels. This could be a clue for the existence of a regulatory mechanism for lipoprotein lipid uptake different from the liver and other tissues.

Current nutritional and drug treatment approaches for hypercholesterolemia do not distinguish between CE and FC in LDL. Together with our previous studies (6-8), the results of the present investigation suggest that the two cholesterol forms present distinct metabolic behavior in pathological conditions and under therapeutic or dietary interventions. CE and FC have also distinct potential for harm, as shown for macrophage apoptosis, which is induced by FC but not CE (35).

The induction of hyperlipidemia and atherosclerosis created a dissociation between the metabolism of the FC and CE components of an artificial lipoprotein model regarding plasma kinetics and hepatic uptake. 


\section{References}

1. Maranhao RC, Roland IA, Toffoletto $O$, Ramires JA, Goncalves RP, Mesquita $\mathrm{CH}$, et al. Plasma kinetic behavior in hyperlipidemic subjects of a lipidic microemulsion that binds to low density lipoprotein receptors. Lipids 1997; 32: 627-633.

2. Sposito AC, Lemos PA, Santos RD, Hueb W, Vinagre CG, Quintella E, et al. Impaired intravascular triglyceride lipolysis constitutes a marker of clinical outcome in patients with stable angina undergoing secondary prevention treatment: a long-term follow-up study. J Am Coll Cardiol 2004; 43: 22252232.

3. Puk CG, Vinagre CG, Bocchi E, Bacal F, Stolf N, Maranhao RC. Plasma kinetics of a cholesterol-rich microemulsion in patients submitted to heart transplantation. Transplantation 2004; 78: 1177-1181.

4. Maranhao RC, Garicochea B, Silva EL, Dorlhiac-Llacer P, Cadena SM, Coelho IJ, et al. Plasma kinetics and biodistribution of a lipid emulsion resembling low-density lipoprotein in patients with acute leukemia. Cancer Res 1994; 54: 46604666.

5. Hirata RD, Hirata $\mathrm{MH}$, Mesquita $\mathrm{CH}$, Cesar TB, Maranhao RC. Effects of apolipoprotein B-100 on the metabolism of a lipid microemulsion model in rats. Biochim Biophys Acta 1999; 1437: 53-62.

6. Santos RD, Hueb W, Oliveira AA, Ramires JA, Maranhao $\mathrm{RC}$. Plasma kinetics of a cholesterol-rich emulsion in subjects with or without coronary artery disease. J Lipid Res 2003; 44: 464-469.

7. Santos RD, Chacra AP, Morikawa A, Vinagre CC, Maranhao $\mathrm{RC}$. Plasma kinetics of free and esterified cholesterol in familial hypercholesterolemia: effects of simvastatin. Lipids 2005; 40: 737-743.

8. Couto RD, Dallan LA, Lisboa LA, Mesquita CH, Vinagre CG, Maranhao RC. Deposition of free cholesterol in the blood vessels of patients with coronary artery disease: a possible novel mechanism for atherogenesis. Lipids 2007; 42: 411418.

9. Maranhao RC, Garicochea B, Silva EL, Llacer PD, Pileggi FJ, Chamone DA. Increased plasma removal of microemulsions resembling the lipid phase of low-density lipoproteins (LDL) in patients with acute myeloid leukemia: a possible new strategy for the treatment of the disease. Braz J Med Biol Res 1992; 25: 1003-1007.

10. Marchese SR, Mesquita $\mathrm{CH}$, Cunha IL. Anacomp program application to calculate $137 \mathrm{C}$ transfer rates in marine organisms and dose in man. J Radioan Nucl Chem 1998; 232: 233236.

11. Folch J, Lees M, Sloane Stanley GH. A simple method for the isolation and purification of total lipides from animal tissues. $J$ Biol Chem 1957; 226: 497-509.

12. Yamazaki S, Mitsunaga T, Furukawa $Y$, Nishida T. Interaction of lecithin-cholesterol acyltransferase with human plasma lipoproteins and with lecithin-cholesterol vesicles. J Biol Chem 1983; 258: 5847-5853.

13. Dobiasova M, Frohlich J. Understanding the mechanism of LCAT reaction may help to explain the high predictive value of LDL/HDL cholesterol ratio. Physiol Res 1998; 47: 387 397.

14. Frohlich J, McLeod R. Lecithin: cholesterol acyltransferase
(LCAT) deficiency syndromes. Adv Exp Med Biol 1986; 201: 181-194.

15. Cheng D, Chang CC, Qu X, Chang TY. Activation of acylcoenzyme A:cholesterol acyltransferase by cholesterol or by oxysterol in a cell-free system. J Biol Chem 1995; 270: 685695.

16. Sturley SL. Molecular aspects of intracellular sterol esterification: the acyl coenzyme A:cholesterol acyltransferase reaction. Curr Opin Lipidol 1997; 8: 167-173.

17. Becker A, Bottcher A, Lackner KJ, Fehringer P, Notka F, Aslanidis $\mathrm{C}$, et al. Purification, cloning, and expression of a human enzyme with acyl coenzyme A:cholesterol acyltransferase activity, which is identical to liver carboxylesterase. Arterioscler Thromb 1994; 14: 1346-1355.

18. Barter P, Kastelein J, Nunn A, Hobbs R. High density lipoproteins (HDLs) and atherosclerosis; the unanswered questions. Atherosclerosis 2003; 168: 195-211.

19. Lundberg BB, Suominen LA. Physicochemical transfer of $\left[{ }^{3} \mathrm{H}\right]$ cholesterol from plasma lipoproteins to cultured human fibroblasts. Biochem J 1985; 228: 219-225.

20. Wang M, Briggs MR. HDL: the metabolism, function, and therapeutic importance. Chem Rev 2004; 104: 119-137.

21. de Grooth GJ, Klerkx AH, Stroes ES, Stalenhoef AF, Kastelein JJ, Kuivenhoven JA. A review of CETP and its relation to atherosclerosis. J Lipid Res 2004; 45: 1967-1974.

22. Gjone E. Familial lecithin cholesterol acyltransferase (LCAT) deficiency. Birth Defects Orig Artic Ser 1982; 18: 423-431.

23. Hoeg JM, Santamarina-Fojo S, Berard AM, Cornhill JF, Herderick EE, Feldman SH, et al. Overexpression of lecithin: cholesterol acyltransferase in transgenic rabbits prevents diet-induced atherosclerosis. Proc Natl Acad Sci U S A 1996; 93: 11448-11453.

24. Lee RG, Kelley KL, Sawyer JK, Farese RV Jr, Parks JS, Rudel LL. Plasma cholesteryl esters provided by lecithin: cholesterol acyltransferase and acyl-coenzyme a:cholesterol acyltransferase 2 have opposite atherosclerotic potential. Circ Res 2004; 95: 998-1004.

25. Brousseau ME, Santamarina-Fojo S, Vaisman BL, Applebaum-Bowden D, Berard AM, Talley GD, et al. Overexpression of human lecithin:cholesterol acyltransferase in cholesterol-fed rabbits: LDL metabolism and HDL metabolism are affected in a gene dose-dependent manner. J Lipid Res 1997; 38: 2537-2547.

26. Brousseau ME, Kauffman RD, Herderick EE, Demosky SJ $\mathrm{Jr}$, Evans W, Marcovina S, et al. LCAT modulates atherogenic plasma lipoproteins and the extent of atherosclerosis only in the presence of normal LDL receptors in transgenic rabbits. Arterioscler Thromb Vasc Biol 2000; 20: 450-458.

27. Maranhao RC, Cesar TB, Pedroso-Mariani SR, Hirata MH, Mesquita $\mathrm{CH}$. Metabolic behavior in rats of a nonprotein microemulsion resembling low-density lipoprotein. Lipids 1993; 28: 691-696.

28. Ho YK, Smith RG, Brown MS, Goldstein JL. Low-density lipoprotein (LDL) receptor activity in human acute myelogenous leukemia cells. Blood 1978; 52: 1099-1114.

29. Brown MS, Goldstein JL. A receptor-mediated pathway for cholesterol homeostasis. Science 1986; 232: 34-47.

30. Nenseter MS, Blomhoff R, Drevon CA, Kindberg GM, Norum $\mathrm{KR}$, Berg T. Uptake of LDL in parenchymal and non-paren- 
chymal rabbit liver cells in vivo. LDL uptake is increased in endothelial cells in cholesterol-fed rabbits. Biochem J 1988; 254: 443-448.

31. Loose-Mitchell DS, Poorman JA, Smith SA, Overturf ML, Morrisett JD, Gotto AM Jr, et al. Cholesterol metabolism in hypercholesterolemia-resistant rabbits. Atherosclerosis 1991; 87: 169-181.

32. Chang S, Borensztajn J. Hepatic lipase function and the accumulation of beta-very-low-density lipoproteins in the plasma of cholesterol-fed rabbits. Biochem J 1993; 293 (Part 3): 745-750.

33. Bocan TM, Mueller SB, Mazur MJ, Uhlendorf PD, Brown EQ, Kieft KA. The relationship between the degree of dietaryinduced hypercholesterolemia in the rabbit and atheroscle- rotic lesion formation. Atherosclerosis 1993; 102: 9-22.

34. Kolodgie FD, Katocs AS Jr, Largis EE, Wrenn SM, Cornhill $J F$, Herderick EE, et al. Hypercholesterolemia in the rabbit induced by feeding graded amounts of low-level cholesterol. Methodological considerations regarding individual variability in response to dietary cholesterol and development of lesion type. Arterioscler Thromb Vasc Biol 1996; 16: 14541464.

35. Kellner-Weibel G, Jerome WG, Small DM, Warner GJ, Stoltenborg JK, Kearney MA, et al. Effects of intracellular free cholesterol accumulation on macrophage viability: a model for foam cell death. Arterioscler Thromb Vasc Biol 1998; 18: 423-431. 